\title{
Universiteit
}

Leiden

The Netherlands

\section{Fiber-connectorized micropillar cavities}

Haupt, F.; Oemrawsingh, S.S.R.; Thon, S.M.; Kim, H.; Kleckner, D.; Ding, D.; ... ;

Bouwmeester, D.

\section{Citation}

Haupt, F., Oemrawsingh, S. S. R., Thon, S. M., Kim, H., Kleckner, D., Ding, D., ...

Bouwmeester, D. (2010). Fiber-connectorized micropillar cavities. Applied Physics Letters, 97, 131113. doi:10.1063/1.3493187

Version: $\quad$ Not Applicable (or Unknown)

License: $\quad$ Leiden University Non-exclusive license

Downloaded from: https://hdl.handle.net/1887/65886

Note: To cite this publication please use the final published version (if applicable). 


\section{Fiber-connectorized micropillar cavities}

Florian Haupt, Sumant S. R. Oemrawsingh, Susanna M. Thon, Hyochul Kim, Dustin Kleckner, Dapeng Ding, Donald J. Suntrup, Pierre M. Petroff, and Dirk Bouwmeester

Citation: Appl. Phys. Lett. 97, 131113 (2010); doi: 10.1063/1.3493187

View online: https://doi.org/10.1063/1.3493187

View Table of Contents: http://aip.scitation.org/toc/apl/97/13

Published by the American Institute of Physics

\section{Articles you may be interested in}

Tuning micropillar cavity birefringence by laser induced surface defects

Applied Physics Letters 95, 251104 (2009); 10.1063/1.3276550

Independent electrical tuning of separated quantum dots in coupled photonic crystal cavities

Applied Physics Letters 99, 161102 (2011); 10.1063/1.3651491

Deterministic nanoassembly of a coupled quantum emitter-photonic crystal cavity system Applied Physics Letters 98, 193103 (2011); 10.1063/1.3571437

Permanent tuning of quantum dot transitions to degenerate microcavity resonances Applied Physics Letters 98, 121111 (2011); 10.1063/1.3569587

Independent tuning of quantum dots in a photonic crystal cavity Applied Physics Letters 95, 243107 (2009); 10.1063/1.3275002

Strong coupling through optical positioning of a quantum dot in a photonic crystal cavity Applied Physics Letters 94, 111115 (2009); 10.1063/1.3103885

\section{Conference Proceedings}

Get $30 \%$ off all print proceedings!
Enter Promotion Code PDF-30 at checkout

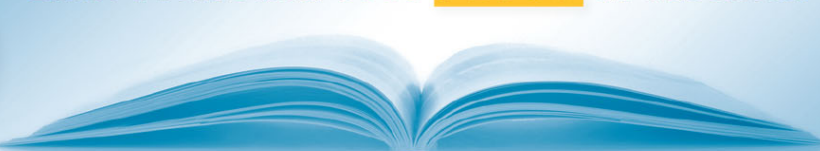




\title{
Fiber-connectorized micropillar cavities
}

Florian Haupt, ${ }^{1, a)}$ Sumant S. R. Oemrawsingh, ${ }^{2, b)}$ Susanna M. Thon, ${ }^{1}$ Hyochul Kim, ${ }^{1, c)}$

Dustin Kleckner, ${ }^{1}$ Dapeng Ding, ${ }^{2}$ Donald J. Suntrup III, ${ }^{1}$ Pierre M. Petroff, ${ }^{3}$ and

Dirk Bouwmeester ${ }^{1,2}$

${ }^{1}$ Department of Physics, University of California at Santa Barbara, Santa Barbara, California 93106, USA

${ }^{2}$ Huygens Laboratory, Leiden University, PO Box 9504, 2300 RA Leiden, The Netherlands

${ }^{3}$ Materials Department, University of California Santa Barbara, Santa Barbara, California 93106, USA and ECE Department, University of California Santa Barbara, Santa Barbara, California 93106, USA

(Received 9 June 2010; accepted 4 September 2010; published online 1 October 2010)

\begin{abstract}
We present a cryogenically compatible method for permanently connecting and coupling a single mode fiber to a single mode of a micropillar cavity with embedded quantum dots (QDs). Efficient coupling of up to $40 \%$ was measured which requires a $300 \mathrm{~nm}$ positioning accuracy that remains preserved during the fiber attachment procedure and during cool-down to $4 \mathrm{~K}$. Fiber coupling, as opposed to conventional free space coupling, makes it possible to connect many such QD-cavity systems within the same cryostat which can interact through an external optical network, facilitating the implementation of hybrid photon/confined-electron schemes for quantum communication and information processing. (C) 2010 American Institute of Physics. [doi:10.1063/1.3493187]
\end{abstract}

Many experimental approaches toward quantum computing are currently under investigation. ${ }^{1-4}$ One promising architecture that could enable robust and scalable quantum computation is based on individual electron spins confined within optically active semiconductor quantum dots (QDs), embedded within optical micropillar cavities, that are matched to external optical modes. ${ }^{5,6}$ Fast subnanosecond optical interactions can be used to manipulate the electron spins such that modest electron spin coherence times on the order of $1 \mu \mathrm{s}$ are sufficient for performing a series of quantum state control and readout operations. ${ }^{7,8}$ Several theoretical schemes have been proposed for hybrid (photon/electronspin) quantum computation and quantum communication using such a system ${ }^{9-12}$ in which all electron spin interactions are mediated through photons and photon-projection measurements. Recently we demonstrated highly efficient free-space mode matching to oxide apertured micropillar devices with integrated charge-tunable InGaAs QDs. ${ }^{13} \mathrm{~A}$ crucial step in making this approach scalable is to replace the external mode matching with integrated fiber coupling. If this is achieved a large number of frequency preselected QDmicropillar structures can be cooled down in a single cryostat and be interconnected and controlled through optical fiber networks. Here we present a method to fiber-couple high-Q micropillar cavities. In contrast to other studies based on mechanical alignment, ${ }^{14,15}$ we demonstrate permanent, precise fiber coupling of a single cavity mode to a single mode fiber by an active alignment procedure.

We use a cavity design that is optimized to produce robust yet small mode-volume micropillar cavities. Etching small trenches in a double GaAs/AlGaAs Bragg reflector structure with a $\lambda / 2$ cavity spacer layer and subsequently partially oxidizing an integrated layer with a high $\mathrm{Al}$ content results in sturdy $30 \mu \mathrm{m}$ size structures [Fig. 1(b)], while

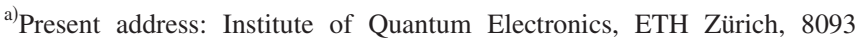
Zürich, Switzerland.

${ }^{\mathrm{b}}$ Electronic mail: oemrawsingh@molphys.leidenuniv.nl.

${ }^{c}$ Present address: Department of Electrical and Computer Engineering, IREAP, University of Maryland, College Park, Maryland 20742, USA.
}

defining an optical mode diameter of as small as $0.5 \mu \mathrm{m} .{ }^{16}$

The first step in the fiber-coupling process is precharacterization of the cavity of interest. We perform room temperature photoluminescence measurements on our sample to determine the resonance frequency of the fundamental micropillar mode.

The second step in the process is prealignment of the optical fiber to the micropillar sample [Figs. 1(a) and 1(b)].

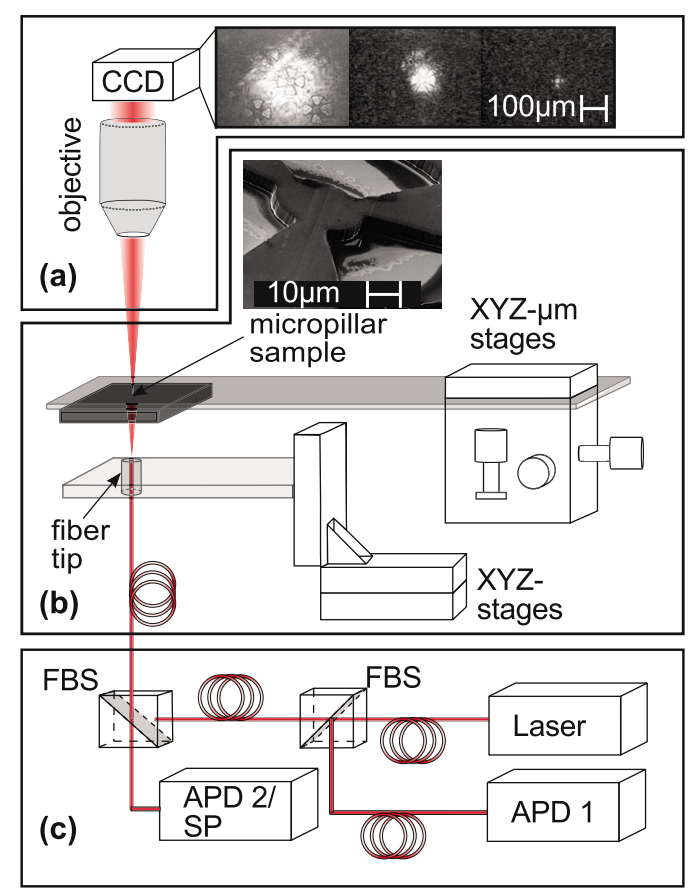

FIG. 1. (Color online) Schematic of the setup: (a) the sample and the fiber tip can be imaged via backside illumination with a $1064 \mathrm{~nm}$ laser through the fiber and a microscope objective (top three images). (b) The oxide apertured micropillar sample (scanning electron microscope image) is mounted on a glass slide which is attached to an XYZ- $\mu \mathrm{m}$ stage for positioning. (c) The sample is probed with a laser either on resonance or pumped above the band gap, coupled through fiber beam splitters (FBS), and the signal is detected using single photon counters (APD 1 and 2) or a CCD camera connected to a spectrometer (SP). 

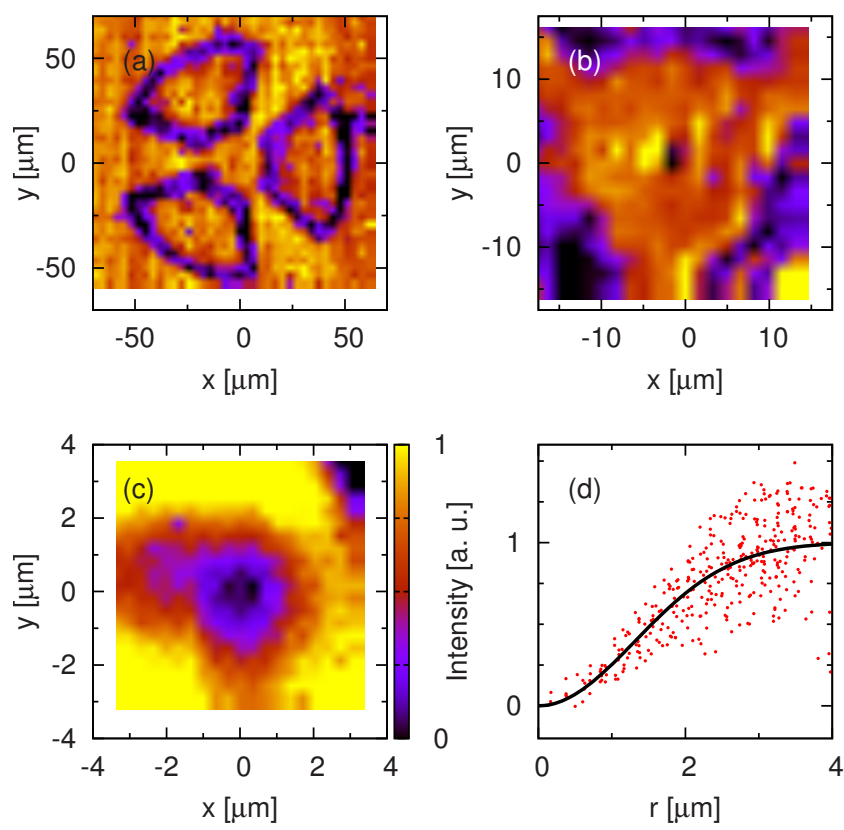

FIG. 2. (Color online) [(a)-(c)] Resonant reflection measurements at room temperature of a micropillar structure with $125 \mu \mathrm{m}$ outer trench diameter with increasing resolution: (a) the full structure, (b) the micropillar itself, and (c) the aperture. (d) Radial plot of (c) and a Gaussian fit, yielding a cavity mode diameter at the surface of $4.0 \pm 1.5 \mu \mathrm{m}$

The chip containing the cavity of interest is mounted on a glass slide attached to a three-axis translation stage with micrometer accuracy. The single-mode fiber (Nufern 780HP) is mounted in a FC/PC bare fiber connector and adjusted so that a small amount sticks out of the ferrule. The fiber is mounted on a motorized three-axis translation stage with a minimal incremental motion of $\sim 50 \mathrm{~nm}$. The sample is illuminated by a $1064 \mathrm{~nm}$ laser that is coupled into the fiber itself and imaged (through the backside of the sample) in transmission by a microscope objective onto a chargecoupled device (CCD). The geometry of the micropillar structures becomes visible due to light scattering at the edges of the trenches. As the single-mode fiber is moved closer to the sample, the area illuminated through the fiber becomes smaller [Fig. 1(a)], allowing us to position the tip of the fiber along the $z$ direction close to the sample with an accuracy equal to the depth of focus $(5-10 \mu \mathrm{m})$. The cavity mode size at the surface of the sample is expected to be in the range of 2-5 $\mu \mathrm{m}$ in diameter depending on the size of the oxidation aperture.

After prealignment, the next step is characterizing the spatial mode of the micropillar cavity. The fundamental cavity mode is probed on-resonance through the fiber with a tunable diode laser at a power of about $0.2 \mathrm{~mW}$, which is frequency-scanned over a range of about $0.2 \mathrm{~nm}$ at $100 \mathrm{~Hz}$. The signal reflected from the micropillar is collected through the same fiber. The reflected signal is detected and normalized to the laser intensity [Fig. 1(c)]. Because the micropillar is a Fabry-Perot resonator, the cavity mode can be identified in a two-dimensional spatial scan of the fiber as an intensity minimum in reflection. The typical result of a spatial scan is shown in Fig. 2. Several spatial scans are performed iteratively at higher resolutions, until the spatial mode of the cavity can be identified with a maximum resolution of 300 $\mathrm{nm}$. The fiber we use has a numerical aperture (N.A.) of 0.13 and a mode field diameter of $5.0 \pm 0.5 \mu \mathrm{m}$ at $850 \mathrm{~nm}$. The

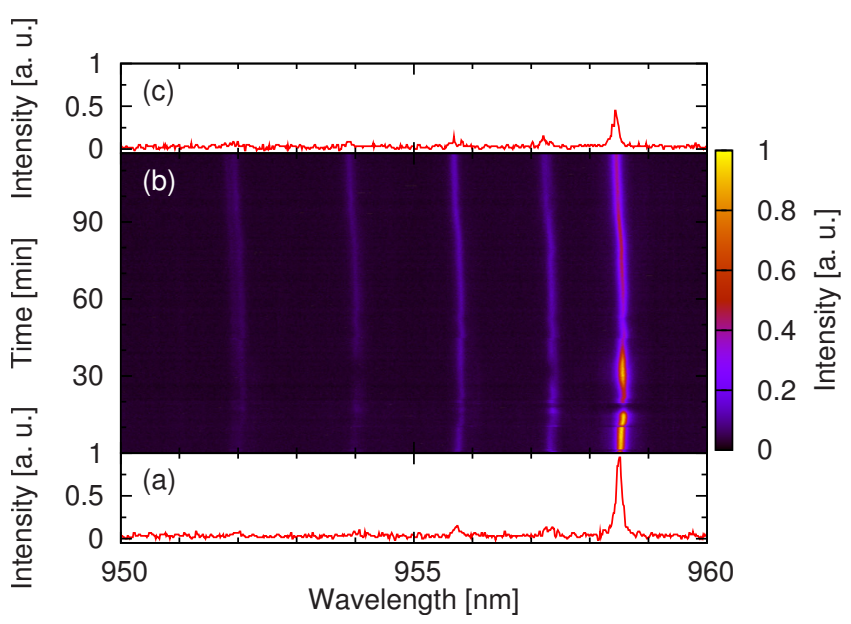

FIG. 3. (Color online) Monitoring the photoluminescence signal from the sample during curing allows for small displacement corrections. (a) Spectrum of a micropillar cavity measured through the fiber before curing. (b) Cavity spectrum as a function of time during the curing process. After about $18 \mathrm{~min}$ a displacement of the fiber was corrected by making a fine position adjustment. (c) Final spectrum after curing.

detected mode is a convolution of the cavity mode and the mode of the fiber. By fitting the detected signal to a Gaussian, as shown in a radial plot in Fig. 2(d), we can extract the average cavity mode diameter at the surface of the sample; for the cavity shown in Fig. 2 the mode diameter is found to be $4.0 \pm 1.5 \mu \mathrm{m}$, which is typical for our devices. Given the mismatch of the fiber and cavity modes, the power transmission coefficient or coupling efficiency is calculated to be in the range of $60 \%-100 \%$. This does not include losses, e.g., due to specular reflections and scattering.

The final step in the connectorization process is permanently gluing the fiber to the surface of the sample. After the scanning steps, the fiber is retracted from the sample, and a small drop of optical epoxy is placed on the sample surface. We use a UV cure epoxy (Norland Optical Adhesive 81) with a low shrinkage volume, a viscosity of $300 \mathrm{cP}$ and a refractive index of about 1.56, which enables durable and efficient bonds. It is crucial that the sample is mounted upside down in order to enable the glue to form a small, radiallysymmetric droplet. The fiber is then aligned by repeating the prealignment and spatial characterization steps. After alignment, the optical epoxy is cured by a UV lamp for about $2 \mathrm{~h}$. It is important to ensure homogeneous curing from all sides. We monitor the coupling efficiency during the curing process by pumping the sample above the band gap of GaAs and observing the photoluminescence signal on a spectrometer (Fig. 3). This allows us to correct in real time for the slight position errors that occur as a result of inhomogeneities in the curing process. After the sample is cured, additional glue is added in order to encapsulate and stabilize the structure for easier removal of the glass slide.

In order to test the robustness of the fiber connectorization procedure, we cooled the sample to cryogenic temperatures using a helium bath cryostat. The sample was mounted at the end of a long vacuum dewar insert rod with integrated optical fiber and electrical connections. The rod was placed in a tube pumped to vacuum and then vented with helium exchange gas to a final pressure of about $10^{-2}$ mbar. We were able to cool the sample to below $10 \mathrm{~K}$ in about $20 \mathrm{~min}$ by placing the tube in a liquid helium dewar. The fiber cou- 


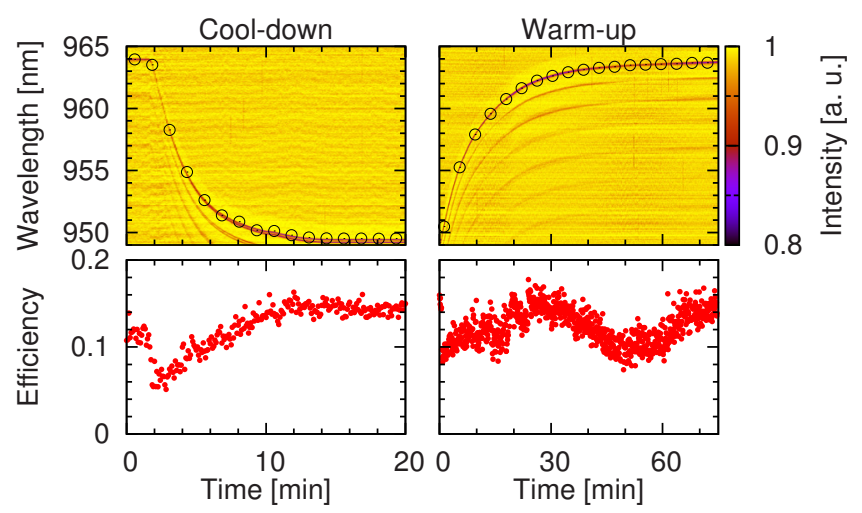

FIG. 4. (Color online) Resonant reflection measurements during cool-down/ warm-up: The sample was cooled to approximately $4 \mathrm{~K}$ in a He dewar insert cryostat, then reheated to room temperature. The upper plots show the reflected signal in false color as a function of wavelength and time, and the lower plots show the calculated coupling efficiency.

pling remained robust for at least two complete cooling and heating cycles. We monitored the shift of the fundamental mode during the cool-down procedure by coupling a broadband LED source centered at $940 \mathrm{~nm}$ into the fiber and observing the reflected signal from the sample. We observed a sharp dip in the spectrum at the fundamental cavity mode frequency and were also able to observe dips due to some of the higher order modes. This is shown in Fig. 4 for both cool-down and warm-up, where the upper plots show how the spectrum (in false color) changes over time, while the lower plots show the coupling efficiency. The depth of the fundamental mode dip (normalized to the bare LED spectrum) gives a measure of the coupling efficiency.

Using the method presented in this paper, we have connectorized eight samples, resulting in coupling efficiencies ranging from $15 \%-40 \%$. The variation in the efficiencies and the deviation from the calculated optimal efficiency for the studied samples and fibers indicate that small changes in the samples, in the fibers, and in the gluing procedure can have significant consequences. Such changes can include variations in the cavity mode waist size and shape, ${ }^{17}$ small angle variations and polishing quality of the fiber tips, and the size (symmetry) of the drop of glue and the UV-curing procedure. We are confident that our $\sim 100 \mathrm{~nm}$ resolution sample fabrication and packaging procedure can be systematically optimized and automated to produce reliable and close to optimal coupling. Our next challenges, in the context of quantum information processing, are the integration of electrical con- tacts to the fiber-coupled micropillars and to achieve twosided fiber coupling to a micropillar cavity so that both the reflection and transmission signals can be collected.

In conclusion, we presented a method of permanently packaging a single mode optical fiber aligned to a single oxide apertured micropillar cavity mode. Our packaging procedure is robust and can be cooled to cryogenic temperatures without a reduction in the coupling efficiency. In principle, we should be able to measure multiple such systems with integrated electrical connections in a single cryostat which can be interconnected and controlled through optical fiber networks, facilitating the implementation of scalable hybrid quantum information schemes.

This work was supported by the National Science Foundation Grant Nos. 0901886, 0622257, and 0804177, the Marie-Curie Grant No. EXT-CT-2006-042580, and an ETH mobility grant.

${ }^{1}$ D. Bouwmeester, A. Zeilinger, and A. K. Ekert, The Physics of Quantum Information (Springer, Berlin, 2001), pp. 133-190.

${ }^{2}$ A. Imamoğlu, D. D. Awschalom, G. Burkard, D. P. DiVincenzo, D. Loss, M. Sherwin, and A. Small, Phys. Rev. Lett. 83, 4204 (1999).

${ }^{3}$ J. Clarke and F. K. Wilhelm, Nature (London) 453, 1031 (2008).

${ }^{4}$ D. Kielpinski, C. Monroe, and D. J. Wineland, Nature (London) 417, 709 (2002).

${ }^{5}$ N. G. Stoltz, M. Rakher, S. Strauf, A. Badolato, D. D. Lofgreen, P. M. Petroff, L. A. Coldren, and D. Bouwmeester, Appl. Phys. Lett. 87, 031105 (2005).

${ }^{6}$ S. Strauf, N. G. Stoltz, M. T. Rakher, L. A. Coldren, P. M. Petroff, and D. Bouwmeester, Nat. Photonics 1, 704 (2007).

${ }^{7}$ J. Berezovsky, M. H. Mikkelsen, N. G. Stoltz, L. A. Coldren, and D. D. Awschalom, Science 320, 349 (2008).

${ }^{8}$ D. Press, T. D. Ladd, B. Zhang, and Y. Yamamoto, Nature (London) 456, 218 (2008).

${ }^{9}$ R. Raussendorf, D. Browne, and H. Briegel, Phys. Rev. A 68, 022312 (2003).

${ }^{10}$ R. Raussendorf and H. J. Briegel, Phys. Rev. Lett. 86, 5188 (2001).

${ }^{11}$ Y. Lim, S. Barrett, A. Beige, P. Kok, and L. Kwek, Phys. Rev. A 73, 012304 (2006).

${ }^{12}$ C. Bonato, F. Haupt, S. S. R. Oemrawsingh, J. Gudat, D. Ding, M. P. van Exter, and D. Bouwmeester, Phys. Rev. Lett. 104, 160503 (2010).

${ }^{13}$ M. T. Rakher, N. G. Stoltz, L. A. Coldren, P. M. Petroff, and D. Bouwmeester, Phys. Rev. Lett. 102, 097403 (2009).

${ }^{14}$ A. Muller, E. B. Flagg, M. Metcalfe, J. Lawall, and G. S. Solomon, Appl. Phys. Lett. 95, 173101 (2009).

${ }^{15}$ X. Xu, F. Brossard, K. Hammura, D. A. Williams, B. Alloing, L. H. Li, and A. Fiore, Appl. Phys. Lett. 93, 021124 (2008).

${ }^{16}$ M. T. Rakher, N. G. Stoltz, L. A. Coldren, P. M. Petroff, and D. Bouwmeester, Appl. Phys. Lett. 93, 091118 (2008).

${ }^{17}$ C. Bonato, D. Ding, J. Gudat, S. Thon, H. Kim, P. M. Petroff, M. P. van Exter, and D. Bouwmeester, Appl. Phys. Lett. 95, 251104 (2009). 